PROCEEDINGS OF THE

AMERICAN MATHEMATICAL SOCIETY

Volume 133, Number 12, Pages 3573-3579

S 0002-9939(05)07897-4

Article electronically published on June 6, 2005

\title{
NECESSARY CONDITIONS FOR SCHATTEN CLASS LOCALIZATION OPERATORS
}

\author{
ELENA CORDERO AND KARLHEINZ GRÖCHENIG
}

(Communicated by David R. Larson)

\begin{abstract}
We study time-frequency localization operators of the form $A_{a}^{\varphi_{1}, \varphi_{2}}$, where $a$ is the symbol of the operator and $\varphi_{1}, \varphi_{2}$ are the analysis and synthesis windows, respectively. It is shown in an earlier paper by the authors that a sufficient condition for $A_{a}^{\varphi_{1}, \varphi_{2}} \in S_{p}\left(L^{2}\left(\mathbb{R}^{d}\right)\right)$, the Schatten class of order $p$, is that $a$ belongs to the modulation space $M^{p, \infty}\left(\mathbb{R}^{2 d}\right)$ and the window functions to the modulation space $M^{1}$. Here we prove a partial converse: if $A_{a}^{\varphi_{1}, \varphi_{2}} \in S_{p}\left(L^{2}\left(\mathbb{R}^{d}\right)\right)$ for every pair of window functions $\varphi_{1}, \varphi_{2} \in \mathcal{S}\left(\mathbb{R}^{2 d}\right)$ with a uniform norm estimate, then the corresponding symbol $a$ must belong to the modulation space $M^{p, \infty}\left(\mathbb{R}^{2 d}\right)$. In this sense, modulation spaces are optimal for the study of localization operators. The main ingredients in our proofs are frame theory and Gabor frames. For $p=\infty$ and $p=2$, we recapture earlier results, which were obtained by different methods.
\end{abstract}

\section{INTRODUCTION}

Localization operators, as studied by Daubechies [4 in 1988, serve as a tool to localize a signal simultaneously in time and frequency (or in phase space). Under different names, such as Anti-Wick operators, Gabor multipliers, Toeplitz operators or wave packets, they have become useful in PDE, in quantum mechanics and quantization, or in signal analysis and optics, and therefore they have been studied extensively. For a sample of diverse contributions and extended references, we refer to $1,3,4,4,18,9,11,12,19,20$.

In 3, localization operators are studied as a part of time-frequency analysis. Such an approach is suggested by the very definition of a localization operator by means of the short-time Fourier transform. Using the function spaces that are naturally associated to the short-time Fourier transform, the so-called modulation spaces, we have obtained the sharpest sufficient conditions for boundedness and Schatten class properties that are known so far [3]. For bounded operators and for Hilbert-Schmidt operators we have also shown that conditions involving modulation spaces are also necessary.

Received by the editors April 7, 2004 and, in revised form, July 12, 2004.

2000 Mathematics Subject Classification. Primary 35S05, 47G30, 46E35, 47B10.

Key words and phrases. Localization operator, modulation space, short-time Fourier transform, Schatten class operator.

The first author was supported in part by the national project MIUR "Analisi Armonica" (coordination by G. Mauceri). 
In this note we answer the following question: What can be said about the symbols of operators $A_{a}^{\varphi_{1}, \varphi_{2}}$ in the Schatten class $S_{p}\left(L^{2}\left(\mathbb{R}^{d}\right)\right)$, when $p \neq 2$ or $p \neq \infty$ ?

Our answer will demonstrate once more that modulation spaces are the appropriate symbol classes for the study of localization operators and that they are in a sense optimal.

The technical innovation in this paper is the use of frame theory, in particular Gabor frames, to study the Schatten class properties of localization operators.

Statement of results. The protagonists of time-frequency analysis are the timefrequency shifts (or phase space translations) defined by

$$
M_{\omega} T_{x} f(t)=e^{2 \pi i \omega t} f(t-x), \quad t, x, \omega \in \mathbb{R}^{d} .
$$

For a fixed non-zero $g \in \mathcal{S}\left(\mathbb{R}^{d}\right)$ (the Schwartz class), the short-time Fourier transform (for short STFT) of $f \in \mathcal{S}^{\prime}\left(\mathbb{R}^{d}\right)$ (the space of tempered distributions), with respect to the window $g$, is given by

$$
V_{g} f(x, \omega)=\left\langle f, M_{\omega} T_{x} g\right\rangle=\int_{\mathbb{R}^{d}} f(t) \overline{g(t-x)} e^{-2 \pi i \omega t} d t .
$$

A time-frequency localization operator $A_{a}^{\varphi_{1}, \varphi_{2}}$ with symbol $a$, analysis window function $\varphi_{1}$, and synthesis window function $\varphi_{2}$ is defined formally by means of the STFT as

$$
A_{a}^{\varphi_{1}, \varphi_{2}} f=\int_{\mathbb{R}^{2 d}} a(x, \omega) V_{\varphi_{1}} f(x, \omega) M_{\omega} T_{x} \varphi_{2} d x d \omega,
$$

whenever the vector-valued integral makes sense. If $\varphi_{1}(t)=\varphi_{2}(t)=e^{-\pi t^{2}}$, then $A_{a}^{\varphi_{1}, \varphi_{2}}$ boils down to the classical Anti-Wick operator and the mapping $a \mapsto A_{a}^{\varphi_{1}, \varphi_{2}}$ is interpreted as a quantization rule [1, 17, 20].

In a weak sense, the definition of $A_{a}^{\varphi_{1}, \varphi_{2}}$ in (3) can be expressed by

$$
\left\langle A_{a}^{\varphi_{1}, \varphi_{2}} f, g\right\rangle=\left\langle a V_{\varphi_{1}} f, V_{\varphi_{2}} g\right\rangle=\left\langle a, \overline{V_{\varphi_{1}} f} V_{\varphi_{2}} g\right\rangle, \quad f, g \in \mathcal{S}\left(\mathbb{R}^{d}\right) .
$$

Using (4) it is easy to see that if $a \in \mathcal{S}^{\prime}\left(\mathbb{R}^{2 d}\right)$ and $\varphi_{1}, \varphi_{2} \in \mathcal{S}\left(\mathbb{R}^{d}\right)$, then $A_{a}^{\varphi_{1}, \varphi_{2}}$ is a well-defined continuous operator from $\mathcal{S}\left(\mathbb{R}^{d}\right)$ to $\mathcal{S}^{\prime}\left(\mathbb{R}^{d}\right)$.

In 3 we have studied in detail the class of localization operators as a multilinear mapping

$$
\left(a, \varphi_{1}, \varphi_{2}\right) \mapsto A_{a}^{\varphi_{1}, \varphi_{2}},
$$

and derived norm estimates when $a, \varphi_{1}$ and $\varphi_{2}$ belong to certain modulation spaces. Modulation space norms measure the time-frequency concentration (phase space distribution) of functions and distributions on the time-frequency plane (on phase space). The unweighted modulation spaces are defined as follows: Given a non-zero window $g \in \mathcal{S}\left(\mathbb{R}^{d}\right), 1 \leq p, q \leq \infty$, the modulation space $M^{p, q}\left(\mathbb{R}^{d}\right)$ consists of all tempered distributions $f \in \mathcal{S}^{\prime}\left(\mathbb{R}^{d}\right)$ such that $V_{g} f \in L^{p, q}\left(\mathbb{R}^{2 d}\right)$ (mixed-norm spaces). The norm on $M^{p, q}$ is given by

$$
\|f\|_{M^{p, q}}:=\left\|V_{g} f\right\|_{L^{p, q}}=\left(\int_{\mathbb{R}^{d}}\left(\int_{\mathbb{R}^{d}}\left|V_{g} f(x, \omega)\right|^{p} d x\right)^{q / p} d \omega\right)^{1 / q} .
$$

If $p=q$, we write $M^{p}$ instead of $M^{p, p}$.

The space $M^{p, q}\left(\mathbb{R}^{d}\right)$ is a Banach space whose definition is independent of the choice of the window $g$. Different non-zero windows $g \in M^{1}$ yield equivalent norms on $M^{p, q}$. This property will be crucial in the sequel, because we will choose a 
suitable window $g$ in estimates of the $M^{p, q}$-norm. Within the class of modulation spaces, one finds several standard function spaces, for instance $M^{2}=L^{2}, M^{1}$ coincides with Feichtinger's algebra $S_{0}\left(\mathbb{R}^{d}\right)$, and using weighted versions, one also finds certain Sobolev spaces and the Shubin classes among the modulation spaces [3, 10. For more background and the general theory of modulation spaces we refer to 6 , 10.

Our main result about modulation spaces and localization operators can be formulated as follows.

Theorem 1. Let $1 \leq p \leq \infty$.

(a) The mapping $\left(a, \varphi_{1}, \varphi_{2}\right) \mapsto A_{a}^{\varphi_{1}, \varphi_{2}}$ is bounded from $M^{p, \infty}\left(\mathbb{R}^{2 d}\right) \times M^{1}\left(\mathbb{R}^{d}\right) \times$ $M^{1}\left(\mathbb{R}^{d}\right)$ into $S_{p}\left(L^{2}\left(\mathbb{R}^{d}\right)\right)$ with a norm estimate

$$
\left\|A_{a}^{\varphi_{1}, \varphi_{2}}\right\|_{S_{p}} \leq B\|a\|_{M^{p, \infty}}\left\|\varphi_{1}\right\|_{M^{1}}\left\|\varphi_{2}\right\|_{M^{1}}
$$

for a suitable constant $B>0$.

(b) Conversely, assume that $A_{a}^{\varphi_{1}, \varphi_{2}} \in S_{p}\left(L^{2}\left(\mathbb{R}^{d}\right)\right)$ for all windows $\varphi_{1}, \varphi_{2} \in M^{1}$ and that there exists a constant $B>0$ depending only on the symbol a such that

$$
\left\|A_{a}^{\varphi_{1}, \varphi_{2}}\right\|_{S_{p}} \leq B\left\|\varphi_{1}\right\|_{M^{1}}\left\|\varphi_{2}\right\|_{M^{1}}, \quad \forall \varphi_{1}, \varphi_{2} \in \mathcal{S}\left(\mathbb{R}^{d}\right) .
$$

Then $a \in M^{p, \infty}$.

While it seems hopeless to find a characterization for $A_{a}^{\varphi_{1}, \varphi_{2}} \in S_{p}$ with a fixed pair of windows $\varphi_{1}, \varphi_{2}$, our main result shows that the condition $a \in M^{p, \infty}$ is optimal for a natural class of windows. Part (a) was already proved in [3] and was shown to include the known conditions in [2, 8, 20]. Part (b) was known only for the cases $p=2$ and $p=\infty[3$. By developing a new method, we are able to fill this gap and prove statement (b) for all values of $p$.

Notation. We define $t^{2}=t \cdot t$, for $t \in \mathbb{R}^{d}$, and $x y=x \cdot y$ is the scalar product on $\mathbb{R}^{d}$. When using the duality $\langle f, g\rangle$ for $f$ in the Schwartz class $\mathcal{S}\left(\mathbb{R}^{d}\right)$ and $g \in \mathcal{S}^{\prime}\left(\mathbb{R}^{d}\right)$ a tempered distribution, the bracket is always understood to extend the inner product $\langle f, g\rangle=\int f(t) \overline{g(t)} d t$ on $L^{2}\left(\mathbb{R}^{d}\right)$.

Throughout the paper, we shall use the notation $A \lesssim B$ to indicate $A \leq c B$ for a suitable constant $c>0$, whereas $A \asymp B$ if $A \leq c B$ and $B \leq k A$, for suitable $c, k>0$.

\section{Some time-FREQUENCY ANALYSIS}

We collect a few facts about the STFT and Gabor frames needed in the sequel. First some properties of the STFT.

Lemma 1. Write $z=\left(z_{1}, z_{2}\right) \in \mathbb{R}^{2 d}, \zeta=\left(\zeta_{1}, \zeta_{2}\right) \in \mathbb{R}^{2 d}$. For $\varphi_{1}, \varphi_{2} \in \mathcal{S}\left(\mathbb{R}^{d}\right)$ and $f, g \in \mathcal{S}^{\prime}\left(\mathbb{R}^{d}\right)$ we have

$$
\begin{aligned}
T_{\left(z_{1}, z_{2}\right)}\left(\overline{V_{\varphi_{1}} f} V_{\varphi_{2}} g\right)(x, \omega) & =\overline{V_{\varphi_{1}}\left(M_{z_{2}} T_{z_{1}} f\right)}(x, \omega) V_{\varphi_{2}}\left(M_{z_{2}} T_{z_{1}} g\right)(x, \omega), \\
M_{\left(\zeta_{1}, \zeta_{2}\right)}\left(\overline{V_{\varphi_{1}} f} V_{\varphi_{2}} g\right)(x, \omega) & =\overline{V_{\varphi_{1}} f(x, \omega)} V_{\left(M_{\zeta_{1}} T_{-\zeta_{2}} \varphi_{2}\right)}\left(M_{\zeta_{1}} T_{-\zeta_{2}} g\right)(x, \omega), \\
M_{\zeta} T_{z}\left(\overline{V_{\varphi_{1}} f} V_{\varphi_{2}} g\right) & =\overline{V_{\varphi_{1}}\left(M_{z_{2}} T_{z_{1}} f\right)} V_{\left(M_{\zeta_{1}} T_{-\zeta_{2}} \varphi_{2}\right)}\left(M_{\zeta_{1}} T_{-\zeta_{2}} M_{z_{2}} T_{z_{1}} g\right) .
\end{aligned}
$$

These formulas are obtained by a minimal modification of formulas (25), (26), and (27) in [3]. 
The STFT of the $d$-dimensional Gaussian $\varphi(x):=e^{-\pi x^{2}}$ is given by 10, Lemma 1.5.2] as

$$
V_{\varphi} \varphi(x, \omega)=2^{-d / 2} e^{-\pi i x \omega} e^{-\frac{\pi}{2}\left(x^{2}+\omega^{2}\right)} .
$$

Gaussians are particularly suited as windows to measure modulation space norms because of the following discrete version of the modulation space norm.

Theorem 2. If $\Phi(x, \omega)=e^{-\pi\left(x^{2}+\omega^{2}\right)}$ and $\alpha \beta<1$, then $F \in M^{p, q}\left(\mathbb{R}^{2 d}\right)$ if and only if

$$
\left\|\left\langle F, M_{\beta n} T_{\alpha k} \Phi\right\rangle_{n, k \in \mathbb{Z}^{2 d}}\right\|_{\ell p, q}\left(\mathbb{Z}^{4 d}\right):=\left(\sum_{n \in \mathbb{Z}^{2 d}}\left(\sum_{k \in \mathbb{Z}^{2 d}}\left|\left\langle F, M_{\beta n} T_{\alpha k} \Phi\right\rangle\right|^{p}\right)^{q / p}\right)^{1 / q}
$$

is finite. Moreover, $\|F\|_{M^{p, q}} \asymp\left\|\left\langle F, M_{\beta n} T_{\alpha k} \Phi\right\rangle_{n, k \in \mathbb{Z}^{2 d}}\right\|_{\ell^{p, q}\left(\mathbb{Z}^{4 d}\right)}$.

Remark. Theorem 2 contains more than meets the eye and is, in fact, a summary of some fundamental results of Gabor analysis. If $p=q=2$, then the norm equivalence $\|F\|_{2} \asymp\left\|\left\langle F, M_{\beta n} T_{\alpha k} \Phi\right\rangle_{n, k \in \mathbb{Z}^{2 d}}\right\|_{2}$ expresses that the collection $\left\{M_{\beta n} T_{\alpha k} \Phi: k, n \in \mathbb{Z}^{2 d}\right\}$ is a Gabor frame for $L^{2}\left(\mathbb{R}^{2 d}\right)$. This is the case if and only if $\alpha \beta<1$ by a deep result of Seip and Wallstén [15, 16. They proved it in dimension $d=1$, but by a tensor product argument the result carries over to higher dimensions. By [7] or [10, Corollary 12.2.6] the Gabor frame $\left\{M_{\beta n} T_{\alpha k} \Phi: k, n \in \mathbb{Z}^{2 d}\right\}$ then extends to a Banach frame for the modulation spaces $M^{p, q}\left(\mathbb{R}^{2 d}\right.$ ) (and also weighted modulation spaces). In particular, the norm equivalence (8) holds.

The norm equivalence (8) is the main tool in the proof of Theorem 1

\section{Necessary Schatten Class COnditions for localization operators}

We can now prove part (b) of Theorem 1. Recall that a bounded (in fact, compact) operator $L$ on a Hilbert space $\mathcal{H}$ belongs to the Schatten class $S_{p}(\mathcal{H})$ if the sequence $\left\{\lambda_{n}: n \in \mathbb{N}\right\}$ of eigenvalues of $\left(L^{*} L\right)^{1 / 2}$, the so-called singular values, belongs to $\ell^{p}$. The norm on $S_{p}$ is given by $\|L\|_{S_{p}}=\|\lambda\|_{p}$ (see the standard references [18, 17]). Equivalently, we may express the norm on $S_{p}(\mathcal{H})$ by

$$
\|L\|_{S_{p}}^{p}=\sup \sum_{j}\left\|L e_{j}\right\|_{\mathcal{H}}^{p}
$$

where the supremum is taken over all orthonormal bases $\left\{e_{j}: j \in \mathcal{J}\right\}$ of $\mathcal{H}$. As a straightforward consequence we obtain the inequality

$$
\left(\sum_{j \in \mathcal{J}}\left|\left\langle L e_{j}, e_{j}\right\rangle\right|^{p}\right)^{1 / p} \leq\|L\|_{S_{p}}
$$

for every orthonormal basis $\left\{e_{j}: j \in \mathcal{J}\right\}$.

This last inequality extends easily to frames as was already observed in [14.

Lemma 3. Let $\left\{b_{j}\right\}_{j \in \mathcal{J}}$ be a frame for $\mathcal{H}$. If $L \in S_{p}(\mathcal{H})$, for $1 \leq p \leq \infty$, then

$$
\left(\sum_{j \in \mathcal{J}}\left|\left\langle L b_{j}, b_{j}\right\rangle\right|^{p}\right)^{1 / p} \lesssim\|L\|_{S_{p}}
$$


Proof. We prove the claim for $p=1$ and $p=\infty$ and then use interpolation.

According to the spectral theorem for compact (non-self-adjoint) operators 13 , Thm. VI.17] for $L \in S_{p}(\mathcal{H})$ there exist two orthonormal sets $\left\{e_{k}: k \in \mathbb{N}\right\}$ and $\left\{f_{k}: k \in \mathbb{N}\right\}$, such that

$$
L f=\sum_{k=1}^{\infty} \lambda_{k}\left\langle f, e_{k}\right\rangle f_{k}
$$

where the $\lambda_{k}$ 's are the singular values of $L$.

Case $p=1$. Since $\left\{b_{j}\right\}$ is a frame, we have $\sum_{j}\left|\left\langle f, b_{j}\right\rangle\right|^{2} \leq D\|f\|_{\mathcal{H}}^{2}$ for all $f \in \mathcal{H}$, and we can estimate as follows:

$$
\begin{aligned}
\sum_{j \in \mathcal{J}}\left|\left\langle L b_{j}, b_{j}\right\rangle\right| & =\sum_{j \in \mathcal{J}}\left|\sum_{k \in \mathbb{N}} \lambda_{k}\left\langle b_{j}, e_{k}\right\rangle\left\langle f_{k}, b_{j}\right\rangle\right| \\
& \leq \sum_{k \in \mathbb{N}} \lambda_{k} \sum_{j \in \mathcal{J}}\left|\left\langle b_{j}, e_{k}\right\rangle\right|\left|\left\langle f_{k}, b_{j}\right\rangle\right| \\
& \leq \sum_{k \in \mathbb{N}} \lambda_{k}\left(\sum_{j \in \mathcal{J}}\left|\left\langle b_{j}, e_{k}\right\rangle\right|^{2}\right)^{1 / 2}\left(\sum_{j \in \mathcal{J}}\left|\left\langle b_{j}, f_{k}\right\rangle\right|^{2}\right)^{1 / 2} \\
& \leq D \sum_{k \in \mathbb{N}} \lambda_{k}\left\|e_{k}\right\|\left\|f_{k}\right\| \\
& =D \sum_{k \in \mathbb{N}} \lambda_{k}=D\|L\|_{S_{1}},
\end{aligned}
$$

Case $p=\infty$. If $L$ is compact or bounded on $\mathcal{H}$, then

$$
\sup _{j \in \mathcal{J}}\left|\left\langle L b_{j}, b_{j}\right\rangle\right| \leq \sup _{j \in \mathcal{J}}\|L\|_{o p}\left\|b_{j}\right\|_{L^{2}}^{2} \leq D\|L\|_{o p} .
$$

Using complex interpolation between $S_{1}$ and the bounded (or compact) operators on $\mathcal{H}$, the estimate follows for all $p \in[1, \infty]$.

Corollary 2. Let $\left\{b_{j}\right\}_{j \in \mathcal{J}}$ be a frame for $\mathcal{H}$. If $T$ is a bounded operator on $\mathcal{H}$ and $L \in S_{p}(\mathcal{H}), 1 \leq p \leq \infty$, then we have

$$
\left(\sum_{j \in \mathcal{J}}\left|\left\langle L b_{j}, T b_{j}\right\rangle\right|^{p}\right)^{1 / p} \lesssim\|L\|_{S_{p}}\|T\|_{o p} .
$$

Proof. We have $\left\langle L b_{j}, T b_{j}\right\rangle=\left\langle T^{*} L b_{j}, b_{j}\right\rangle$. Since $S_{p}$ is an operator ideal, we have $\left\|T^{*} L\right\|_{S_{p}} \leq\left\|T^{*}\right\|_{o p}\|L\|_{S_{p}}=\|T\|_{o p}\|L\|_{S_{p}}$, and so we may apply Lemma 3 to $T^{*} L$.

Remark. For the above estimates we have only used that $\left\{b_{j}\right\}$ is a Bessel sequence. Proof of Theorem 1. The idea is to compute the $M^{p, \infty}$-norm of the symbol $a$ of the operator $A_{a}^{\varphi_{1}, \varphi_{2}}$ by using a Gabor frame. We choose the Gaussian window $\Phi(x, \omega)=2^{-d} e^{-\pi\left(x^{2}+\omega^{2}\right)}$ and $0<\alpha, \beta<1$. Then the set $\left\{M_{\beta n} T_{\alpha k} \Phi\right\}_{k, n \in \mathbb{Z}^{2 d}}$ is a Gabor frame, and we can estimate the $M^{p, \infty}\left(\mathbb{R}^{2 d}\right)$-norm of $a$ by (8) of Theorem 2 in the form

$$
\|a\|_{M^{p, \infty}\left(\mathbb{R}^{2 d}\right)} \asymp\left\|\left\langle a, M_{\beta n} T_{\alpha k} \Phi\right\rangle_{n, k \in \mathbb{Z}^{2 d}}\right\|_{\ell^{p, \infty}\left(\mathbb{Z}^{4 d}\right)} .
$$

Therefore, we focus our attention on the Gabor coefficients in (11). Using (7), we can write $\Phi$ as

$$
\Phi(x, \omega)=2^{-d} e^{-\pi\left(x^{2}+\omega^{2}\right)}=V_{\varphi} \varphi(x, \omega) \overline{V_{\varphi} \varphi(x, \omega)} .
$$


Next, with $k=\left(k_{1}, k_{2}\right), n=\left(n_{1}, n_{2}\right) \in \mathbb{Z}^{2 d}$ and Lemma 1, we write the timefrequency shifts of $\Phi$ as the product of two STFTs on $\mathbb{R}^{d}$ :

$$
\begin{aligned}
& M_{\beta n} T_{\alpha k} \Phi(x, \omega)=M_{\left(\beta n_{1}, \beta n_{2}\right)} T_{\left(\alpha k_{1}, \alpha k_{2}\right)}\left(V_{\varphi} \varphi \overline{V_{\varphi} \varphi}\right)(x, \omega) \\
& \quad=V_{\left(M_{\beta n_{1}} T_{-\beta n_{2}} \varphi\right)}\left(M_{\beta n_{1}} T_{-\beta n_{2}} M_{\alpha k_{2}} T_{\alpha k_{1}} \varphi\right)(x, \omega) \overline{V_{\varphi}\left(M_{\alpha k_{2}} T_{\alpha k_{1}} \varphi\right)(x, \omega)} .
\end{aligned}
$$

Now we substitute this formula into the weak definition of localization operators in (4) and we obtain the following decisive relation between the Gabor coefficients of the symbol $a$ and the localization operator $A_{a}^{\varphi_{1}, \varphi_{2}}$ :

$$
\left\langle a, M_{\beta n} T_{\alpha k} \Phi\right\rangle=\left\langle A_{a}^{\varphi, M_{\beta n_{1}} T_{-\beta n_{2}} \varphi}\left(M_{\alpha k_{2}} T_{\alpha k_{1}} \varphi\right), M_{\beta n_{1}} T_{-\beta n_{2}} M_{\alpha k_{2}} T_{\alpha k_{1}} \varphi\right\rangle .
$$

Consequently, the $M^{p, \infty}$-norm of $a$ can be recast as

$$
\begin{aligned}
& \|a\|_{M^{p, \infty}} \asymp\left\|\left\langle a, M_{\beta n} T_{\alpha k} \Phi\right\rangle_{n, k \in \mathbb{Z}^{2 d}}\right\|_{\ell^{p, \infty}\left(\mathbb{Z}^{4 d}\right)} \\
& \quad=\sup _{n \in \mathbb{Z}^{2 d}}\left(\sum_{k \in \mathbb{Z}^{2 d}}\left|\left\langle a, M_{\beta n} T_{\alpha k} \Phi\right\rangle\right|^{p}\right)^{1 / p} \\
& \quad=\sup _{n_{1}, n_{2} \in \mathbb{Z}^{d}}\left(\sum_{k_{1}, k_{2} \in \mathbb{Z}^{d}}\left|\left\langle A_{a}^{\varphi, M_{\beta n_{1}} T_{-\beta n_{2}} \varphi}\left(M_{\alpha k_{2}} T_{\alpha k_{1}} \varphi\right), M_{\beta n_{1}} T_{-\beta n_{2}} M_{\alpha k_{2}} T_{\alpha k_{1}} \varphi\right\rangle\right|^{p}\right)^{1 / p},
\end{aligned}
$$

where $n=\left(n_{1}, n_{2}\right), k=\left(k_{1}, k_{2}\right) \in \mathbb{Z}^{2 d}$. Since $\varphi \in \mathcal{S}\left(\mathbb{R}^{d}\right)$, the occurring localization

operators $A_{a}^{\varphi, M_{\beta n_{1}} T_{-\beta n_{2}} \varphi}$ are all in $S_{p}\left(L^{2}\left(\mathbb{R}^{d}\right)\right)$ and by hypothesis they satisfy the uniform estimate

$$
\sup _{\left(n_{1}, n_{2}\right) \in \mathbb{Z}^{2 d}}\left\|A_{a}^{\varphi,\left(M_{\beta n_{1}} T_{-\beta n_{2}} \varphi\right)}\right\|_{S_{p}} \lesssim \sup _{\left(n_{1}, n_{2}\right) \in \mathbb{Z}^{2 d}}\|\varphi\|_{M^{1}}\left\|M_{\beta n_{1}} T_{-\beta n_{2}} \varphi\right\|_{M^{1}}=\|\varphi\|_{M^{1}}^{2},
$$

where we have used the fact that time-frequency shifts are isometries on $M^{1}$ [10, Theorem 11.3.5].

Furthermore, for fixed $n=\left(n_{1}, n_{2}\right) \in \mathbb{Z}^{2 d}$, the right-hand side of (13) has exactly the form discussed in Corollary 2, Since $\alpha<1$, the set $\left\{M_{\alpha k_{2}} T_{\alpha k_{1}} \varphi: k_{1}, k_{2} \in\right.$ $\left.\mathbb{Z}^{d}\right\}$ is a (Gabor) frame for $L^{2}\left(\mathbb{R}^{d}\right)$. Choosing this frame and $T=M_{\beta n_{1}} T_{-\beta n_{2}}$ in Corollary 2, we obtain the desired estimate

$$
\begin{aligned}
\|a\|_{M^{p, \infty}} & \asymp \sup _{n_{1}, n_{2}}\left\|\left\langle A_{a}^{\varphi, M_{\beta n_{1}} T_{-\beta n_{2}} \varphi}\left(M_{\alpha k_{2}} T_{\alpha k_{1}} \varphi\right), M_{\beta n_{1}} T_{-\beta n_{2}} M_{\alpha k_{2}} T_{\alpha k_{1}} \varphi\right\rangle_{k_{1}, k_{2}}\right\|_{\ell^{p}} \\
& \lesssim \sup _{n_{1}, n_{2}}\left\|A_{a}^{\varphi, M_{\beta n_{1}} T_{-\beta n_{2}} \varphi}\right\|_{S_{p}} \lesssim\|\varphi\|_{M^{1}}^{2} .
\end{aligned}
$$

Thus we have shown that $a \in M^{p, \infty}\left(\mathbb{R}^{2 d}\right)$, and the proof is finished.

\section{REFERENCES}

1. F. A. Berezin. Wick and anti-Wick symbols of operators. Mat. Sb. (N.S.), 86(128):578-610, 1971. MR0291839 (45:929)

2. P. Boggiatto and E. Cordero. Anti-Wick quantization of tempered distributions. Proc. of 3rd ISAAC Congress 2001, Berlin. Eds. Begehr, Gilbert, Wong, I, 655-662, 2003. MR2032736 (2004k:35431)

3. E. Cordero and K. Gröchenig. Time-frequency analysis of Gabor localization operators. J. Funct. Anal., 205(1), 107-131, 2003. MR2020210 (2004j:47100)

4. I. Daubechies. Time-frequency localization operators: a geometric phase space approach. IEEE Trans. Inform. Theory, 34(4):605-612, 1988. MR0966733 
5. F. De Mari, H. G. Feichtinger, and K. Nowak. Uniform eigenvalue estimates for timefrequency localization operators. J. London Math. Soc. (2), 65(3):720-732, 2002. MR.1895743 (2003c:47048)

6. H. G. Feichtinger. Modulation spaces on locally compact abelian groups. In Proceedings of "International Conference on Wavelets and Applications" 2002, Chennai, India. Updated version of a technical report, University of Vienna, 1983.

7. H. G. Feichtinger and K. Gröchenig. Gabor frames and time-frequency analysis of distributions. J. Funct. Anal., 146(2):464-495, 1997. MR1452000(98k:42041)

8. H. G. Feichtinger and K. Nowak. A First Survey of Gabor Multipliers. In H. G. Feichtinger and T. Strohmer, editors, Advances in Gabor Analysis. Birkhäuser, Boston, 2002. MR 1955929 (2004e:42003)

9. G. B. Folland. Harmonic Analysis in Phase Space. Princeton Univ. Press, Princeton, NJ, 1989. MR0983366 (92k:22017)

10. K. Gröchenig. Foundations of time-frequency analysis. Birkhäuser Boston Inc., Boston, MA, 2001. MR:1843717 (2002h:42001)

11. R. Howe. Quantum mechanics and partial differential equations. J. Funct. Anal., 38(2):188254, 1980. MR0587908 (83b:35166)

12. J. Ramanathan and P. Topiwala. Time-frequency localization via the Weyl correspondence. SIAM J. Math. Anal., 24(5):1378-1393, 1993. MR1234023 (95b:94012)

13. M. Reed and B. Simon. Methods of modern mathematical physics. I. Academic Press Inc. [Harcourt Brace Jovanovich Publishers], New York, second edition, 1980. MR.0751959 (85e:46002)

14. R. Rochberg and S. Semmes. Nearly weakly orthonormal sequences, singular value estimates, and Calderon-Zygmund operators. J. Funct. Anal., 86(2):237-306, 1989. MR1021138 (90k:47047)

15. K. Seip. Density theorems for sampling and interpolation in the Bargmann-Fock space. I. J. Reine Angew. Math., 429:91-106, 1992. MR.1173117 (93g:46026a)

16. K. Seip and R. Wallstén. Density theorems for sampling and interpolation in the BargmannFock space. II. J. Reine Angew. Math., 429:107-113, 1992. MR1173118 (93g:46026b)

17. M. A. Shubin. Pseudodifferential Operators and Spectral Theory. Springer-Verlag, Berlin, second edition, 2001. Translated from the 1978 Russian original by Stig I. Andersson. MR 1852334 (2002d:47073)

18. B. Simon. Trace ideals and their applications. Cambridge University Press, Cambridge, 1979. MR 0541149 (80k:47048)

19. J. Toft. Modulation spaces and pseudodifferential operators. J. Funct. Anal., 207(2):399-429, 2004. MR2032995 (2004j:35312)

20. M. W. Wong. Wavelets Transforms and Localization Operators, volume 136 of Operator Theory Advances and Applications. Birkhauser, 2002. MR.1918652 (2003i:42003)

Department of Mathematics, Politecnico of Torino, Corso Duca degli Abruzzi 24, 10129 TORINO, ITALY

E-mail address: cordero@dm.unito.it

Institute of Biomathematics and Biometry, GSF - National Research Center for Environment and Health, Ingolstädter Landstrasse 1, 85764 Neuherberg, Germany

E-mail address: karlheinz.groechenig@gsf.de 